\title{
Dissection of a Limb of a Vertebral Artery Fenestration: Case Report
}

\author{
David Nicola, Cheemun Lum, Christine De Meulemeester
}

Keywords: Ataxia, cerebral aneurysms, diagnostic neuroradiology, dissection, dysphagia, headache, magnetic resonance imaging, vascular malformations, stroke imaging

doi:10.1017/cjn.2015.329

Can J Neurol Sci. 2016; 43: 329-331

Fenestrations of vertebral and basilar arteries are considered to be rare, congenital malformations observed in angiographic and autopsy studies. They are vascular abnormalities having two different lumens and endothelium layers, sharing either the same or separate adventitia layers. Basilar artery fenestration is an uncommon developmental anomaly occurring in $1 \%$ to $5 \%$ of autopsy specimens, ${ }^{1}$ Vertebrobasilar junction fenestration is considered to be a subtype of fenestration of the proximal basilar artery. Association of a saccular cerebral aneurysm at the fenestration has been well documented. However, dissection of a limb of the vertebral artery fenestration is extremely rare. We describe a case of dissection of the left limb of a left vertebral artery fenestration that initially presented itself as a suspected vertebrobasilar aneurysm.

\section{Case Report}

A 55-year-old man awoke suddenly from sleep with a sudden onset, severe headache behind the left eye lasting approximately ten minutes. The headache was associated with ataxia, diaphoresis, and vomiting. He also recalls an unusual symptom of dysphagia, which lasted approximately ten minutes. All symptoms resolved spontaneously within 15 minutes. He recalls having a similar headache one year ago, which resolved spontaneously. The patient is a single, marine engineer, with a history of hypertension that was managed with olmesartan at the time. He does not drink or smoke and has no significant personal or family history of cardiac or cerebrovascular disease.

By the time the patient arrived at the local emergency room, he was asymptomatic. He was evaluated for a possible cardiac cause and was discharged that evening without further investigations secondary to unremarkable findings. After investigating his symptoms on the Internet and becoming concerned he had a cerebral aneurysm, the patient presented to the emergency department at our institution three days later. A lumbar puncture (LP) showed acellular cerebrospinal fluid (CSF) without xanthochromia and a computed tomography (CT) scan of the head without contrast was normal.

At this stage, it was appropriate to identify the cause for the patient's dysphagia, which was presumably due to transient ischemia to the lateral medulla (nuclei of cranial nerves IX and X). Thus, the differential diagnosis of a thunderclap headache with associated ataxia, vomiting and dysphagia would include the following:

- aneurysm-related subarachnoid hemorrhage (SAH), with vasospasm resulting in focal cerebral ischemia;
- reversible cerebral vasoconstriction syndrome (RCVS), with or without associated SAH and focal cerebral ischemia;

- artery dissection with focal cerebral ischemia;

- occlusive atherosclerotic thromboembolism with focal cerebral ischemia and;

- migraine with brainstem aura.

Although migraine with brainstem aura can present suddenly and be associated with focal neurological signs and symptoms, dysphagia would be an unusual symptom in relative isolation. Given a negative LP and a CT scan that was negative for $\mathrm{SAH}$, the next appropriate step would be imaging with either computed tomography angiography (CTA) or magnetic resonance angiography (MRA) to assess for RCVS, dissection, thromboembolism or a symptomatic aneurysm.

The CTA of the head and neck was interpreted as an aneurysm measuring approximately $5.5 \mathrm{~mm} \times 2.6 \mathrm{~mm}$ at the vertebrobasilar junction (Figure A). The right vertebral artery was dominant. The patient was referred to the neurointerventional clinic for consideration of endovascular management of the suspected aneurysm. Due to its atypical distally tapered shape and location, magnetic resonance imaging (MRI) was ordered.

A 3 T MRI including time-of-flight (TOF), high-resolution wallimaging pre- and post-contrast images was performed. The TOF images demonstrated the suspected aneurysm however, distally, there was evidence of high-T1 signal suspicious for intramural hematoma within a dissection flap (Figure B and C). High-resolution axial T2 wall-imaging showed an abnormal flow-void converging towards the basilar artery, consistent with a subacute dissection within one limb of a vertebrobasilar fenestration (Figure D). The MRI of the brain parenchyma was normal with no evidence of stroke.

Given the clinical presentation and MRI findings, a cerebral angiogram was performed. This confirmed that the previously suspected aneurysm was in fact the patent proximal portion of a fenestration limb. Distally, there was mild irregularity of the basilar artery (Figure $\mathrm{E}$ and F). The presence of distal tapering of the suspected aneurysm on CTA in combination with the patient's initial presentation, MRI and angiographic findings, suggested that dissection of a portion of the left limb of a vertebrobasilar fenestration was likely the underlying cause, rather than other etiologies such as RCVS or

\footnotetext{
From the Ottawa Hospital Research Institute-University of Ottawa.

Received April 1, 2015. Final Revisions Submitted August 12, 2015.

Correspondence to: Cheemun Lum, The Ottawa Hospital, Civic Campus, Department of Medical Imaging-Neuroradiology section, 1053 Carling Avenue, Ottawa, ON, Canada, K1Y 4E9. Email: chlum@ottawahospital.on.ca
} 
atherosclerotic occlusion. These entities would not typically portray this clinical and radiological picture. With lingering evidence of slight irregularity of the left lateral wall of the basilar artery and the absence of clinical or radiological concern for $\mathrm{SAH}$, the patient was started on low-dose ASA $81 \mathrm{mg}$ daily for thrombosis prophylaxis. His blood pressure was monitored and managed, with a targeted blood pressure of less than 135/85. He had no further headaches or transient ischemic attacks. Six months after initial presentation, repeat MRI/MRA demonstrated similar imaging findings, with no evidence of recanalization of the dissected limb of the fenestration.

\section{Discussion}

Vertebral artery fenestration and vertebral artery dissection have both been described independently, with incidence of approximately $0.23-1.95 \%$ (at angiography) and 1.1 per 100,000 , respectively. ${ }^{2}$ Dissection of a limb of a vertebral artery fenestration is thus extremely rare and has never been reported.

Fenestration or a segmental unfused artery is considered to be an anomaly due to the persistence of a vascular anastomotic channel normally present only in the embryo. ${ }^{3}$ According to Padget's description, at the 32 days of embryonic stage, plexiform and longitudinal anastomotic connections develop between the cervical intersegmental arteries that arise from the primitive dorsal aortae. ${ }^{3}$ The primitive vertebral arteries are formed following conversion of the anastomoses to a single lumen and obliteration of the proximal or aortal ends of the first six cervical intersegmental arteries. ${ }^{3}$ The seventh intersegmental artery will remain and form the origin of the vertebral and subclavian arteries. ${ }^{3}$ Fenestration of the vertebral artery is due to a failure of the plexiform anastomoses to involute into a single vascular channel. ${ }^{3}$

Vertebral artery dissection (VAD) is a tear of the intima of the vertebral artery. The intramural hematoma can arise spontaneously or as a result of trauma. Spontaneous cases are thought to be caused by intrinsic factors that weaken the arterial wall. ${ }^{2}$ Only a very small proportion $(1-4 \%)$ has a clear underlying connective tissue disorder, such as Ehlers-Danlos syndrome type 4 or Marfan's syndrome. ${ }^{2}$

Intramural hemorrhage can evolve in a variety of ways: the hematoma may be asymptomatic; or, if the dissection is subintimal, the hematoma may partially or completely occlude the artery or one of its branches possibly resulting in infarction of the brainstem, cerebellum or, rarely, the spinal cord. ${ }^{4}$ Subadventitial dissections tend to cause pseudoaneurysmal dilatation of the vertebral artery and may compress adjacent neurologic structures. ${ }^{4}$ The intimal disruption and low flow states that arise in VAD create a thrombogenic milieu in which emboli may form and propagate distally resulting in transient ischemia or infarction. ${ }^{4}$ If the VAD ruptures through the adventitia, it may cause sub-arachnoid haemorrhage. On imaging, VAD may present as abnormal wall thickening, aneurysmal dilatation, an intimal flap or occlusion. ${ }^{5}$ At MRI, a dissection typically demonstrates crescentic intramural hematoma on $\mathrm{T} 1$ and $\mathrm{T} 2$ weighted images. ${ }^{5}$ Dissections may recanalize, usually within six months after the onset of symptoms, with the vessel lumen returning to a normal appearance after absorption of the wall hematoma. ${ }^{5}$ However, some cases may remain occluded and display minimal radiological changes on follow-up.

The patient initially had a CTA performed and a diagnosis of a vertebrobasilar junction aneurysm was made. The atypical shape and location of the suspected aneurysm and the patient's clinical presentation prompted further MRI and angiographic imaging.
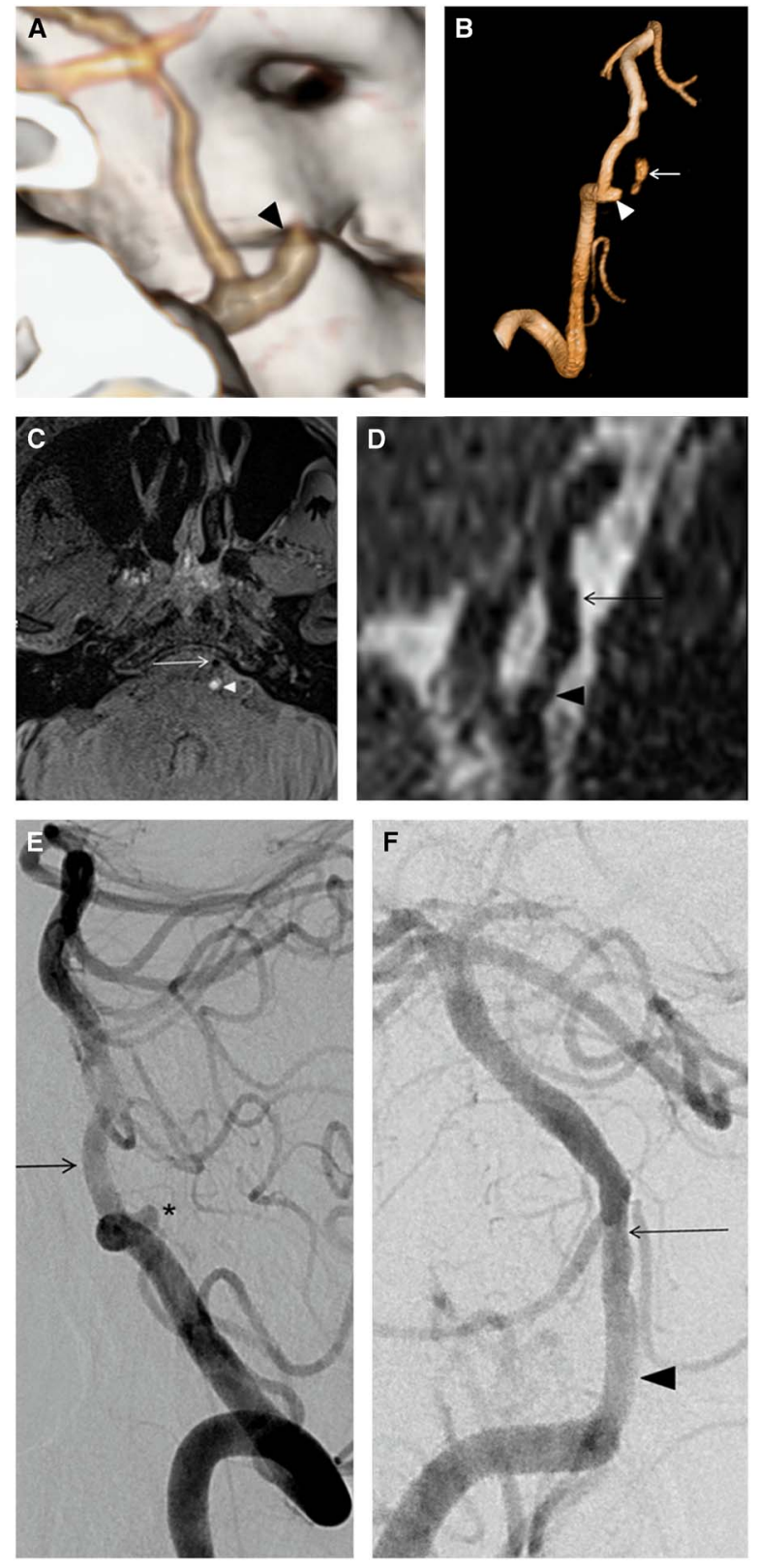

Figure: Left vertebral artery fenestration and left limb dissection mimicking a vertebrobasilar artery aneurysm. (A) CTA 3D reconstruction of left vertebral artery shows distal tapering, a location and appearance atypical for an aneurysm at the vertebrobasilar junction (arrowhead). (B) TOF MRA 3D reconstruction of left vertebral artery shows the suspected aneurysm (arrowhead) and intramural hematoma in the dissection flap (arrow). (C) High-resolution T1-weighted wall image without contrast demonstrates flow void of patent right limb (arrow) and subacute intramural hematoma within the occluded left limb (white arrowhead) of the fenestration. (D) Curved multiplanar reformatted high-resolution sagittal T2-weighted image demonstrates outline of the left limb of the fenestration; the proximal flow void represents the suspected aneurysm (arrowhead) seen on CTA, while the distal portion (arrow) is seen as dark T2 signal from the subacute dissection. (E) Lateral image from right vertebral artery catheter angiogram demonstrates the patent right limb (arrow) and occluded proximal portion of the left limb of the fenestration mimicking a suspected aneurysm (asterisk). (F) AP image of right vertebral artery injection catheter angiogram shows irregular basilar artery wall distally (arrow) and proximal patent left fenestration limb (arrowhead). 
The appearance of a suspected aneurysm on CTA was felt to be secondary to opacification of the patent proximal $1 / 3$ of a fenestration limb. The un-opacified portion of the fenestration, which was not directly visible on CTA, matched the location of focal intramural hematoma on T1-weighted sequences and the hypointense signal on the T2-weighted images. In addition, the high-resolution sagittal reformatted T2-weighted images confirmed the distal $2 / 3$ of the fenestration limb. Arrival at the diagnosis of dissection within a fenestration limb was deduced by careful comparison of the images. Apparent occlusion in the shape of an atypical aneurysm on CTA, as well as focal intramural hematoma on $\mathrm{T} 1$ and dark $\mathrm{T} 2$ signal, all in the same location, favours dissection rather than occlusive thromboembolism. We cannot be entirely certain that the patient's presentation was not the result of atherosclerotic occlusion of the fenestration limb. However, the acute onset of headache and tapered appearance of suspected aneurysm reinforces the diagnosis of dissection. The combination of these elements, as well as subsequent cerebral angiographic imaging confirmed that the previously suspected aneurysm was in fact the patent proximal portion of a fenestration limb, which was most likely occluded by dissection.

In summary, we present a case of dissection within a limb of a vertebral artery fenestration mimicking an intracranial aneurysm. The diagnosis was established by correlating the patient's clinical presentation with imaging from CTA, MRI and catheter angiography.

\section{Disclosures}

David Nicola, Cheemun Lum and Christine De Meulemeester do not have anything to disclose.
This research received no specific grant from any funding agency in the public, commercial, or not-for-profit sectors.

\section{Statement of Authorship}

David Nicola: design, conceptualization of the study, analysis and interpretation of the data, drafting and revising the manuscript. Dr. Lum: design, conceptualization of the study, analysis and interpretation of the data, drafting and revising the manuscript. Dr. De Meulemeester: design, conceptualization of the study, analysis and interpretation of the data, drafting and revising the manuscript.

\section{REFERENCES}

1. Zhang QJ, Kobayashi S, Gibo H, Hongo K. Vertebrobasilar junction fenestration associated with dissecting aneurysm of intracranial vertebral artery. Stroke. 1994;25:1273-5.

2. Kim YK, Schulman S. Cervical artery dissection: pathology, epidemiology and management. Thromb. Res. 2009;123: 810-21.

3. Tseng YC, Hsu HL, Lee TH, Wang LJ, Wong YC, Chen CJ. Fenestration of the vertebral artery at the lower cervical segment: imaging findings and literature review. Eur J Radiol Extra. 2004:49:37-40

4. Lleva P, Ahluwalia BS, Marks S, Sahni R, et al. Traumatic and spontaneous carotid and vertebral artery dissection in a level 1 trauma center. J Clin Neurosci. 2012;19:1112-4.

5. Lum C, Chakraborty S, Schlossmacher M, et al. Vertebral artery dissection with a normal-appearing lumen at multisection CT angiography: the importance of identifying wall hematoma. AJNR Am J Neuroradiol. 2009;30:787-9. 